\title{
A Comparison Between the Macroscopic Anatomy of the Cecum in Laboratory Rat and Guinea Pig
}

\author{
Florin Gheorghe STAN \\ Department of Comparative Anatomy, Faculty of Veterinary Medicine, \\ University of Agricultural Sciences and Veterinary Medicine, Cluj Napoca, Romania \\ *corresponding author: florin.stan@usamvcluj.ro
}

Bulletin UASVM Veterinary Medicine 75(1)/2018

Print ISSN 1843-5270; Electronic ISSN 1843-5378

doi:10.15835/buasvmcn-vm:002817

\begin{abstract}
Controlled food and maintenance conditions cause both functional and anatomical adaptations of digestive system. The rat and guinea pig, used both as experimental models and as pets, shared common controlled environmental conditions. The two species were exposed during the generation to artificial conditions rather that natural ones. The effects of these conditions on the macroscopic anatomy of the cecum have been documented.
\end{abstract}

Keywords: anatomy, cecum, guinea pig, laboratory rat

\section{Introduction}

The cecum is a separate entity of of the intestine in all mammals. Strategically placed between the small and large intestine, its macroscopic anatomy is direct related with the specific functional importance. In this regard, a comparition between the two related species was made in order to highlight the differences and similarities of macroscopic anatomy.

\section{Materials and methods}

The topography, macroscopic anatomy and the mesenterial connection of the cecum, in ten laboratory rats and ten guinea pigs were described and documented.

\section{Results and discussions}

The rat cecum shows variable topography being located in the right side of the abdominal cavity or midventrally into the abdominal cavity, having a transversal orientation (Figure 1). Under conditions of fullness, the rat cecum fills the space between the cranial abdominal organs (liver, stomach, jejunum) and reproductive organs. The rat cecum had a comma shape, was not sacculated and shows no haustra. The ampula ceci is well delineated, having a small constriction at the delineation with the corpus ceci. Also, the exit of the colon is marked by a slight constriction (Figure 2). The ileum and the colon have separate jonctions with the ampula ceci. External appearance of the corpus ceci is smooth and there is no external delineation between the corpus and the apex ceci. In the guinea pig the cecum occupied most of the ventral abdominal cavity, having a more caudal topography compared with the rat (Figure 1). The large, semicircular, thin-walled cecum has numerous lateral haustra and a sacculated appearance. The presence of three white muscular bands along its length, with medial, lateral and ventral disposition lead to the formation of haustra. The apex ceci was les delineated than in the rat, and the two jounctions, namely the ileocecal junction and cecocolic junction was at little distance one from another. The corpus ceci was larger compared with the rat cecum, and exernally the apex ceci show no transition zone between the corpus and apex (Figure 2).The cecal appendix was absent in both species (Stan, 2014). 

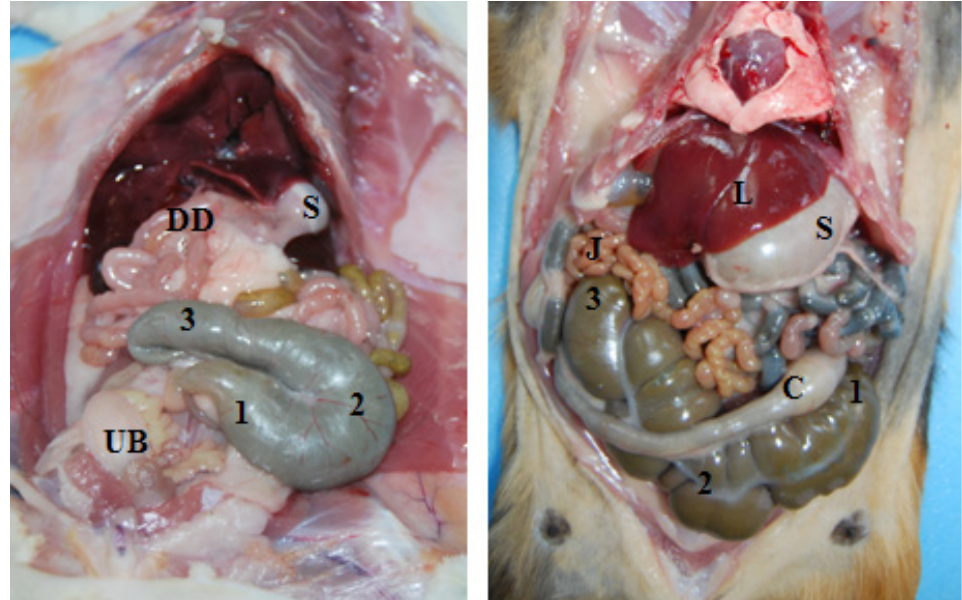

Figure 1. The cecum topography in rat - left and in guinea pig - right.

1 - Ampula ceci; 2 - Corpus ceci; 3 - Apex ceci. In rat the cecum occupied the left part of the ventral abdominal cavity and in guinea pig has a more central and caudal topography. L - liver; S - stomach; DD - descending duodenum; J - jejunum; C - ascendant ansa of the colon; UB - Urinary bladder.
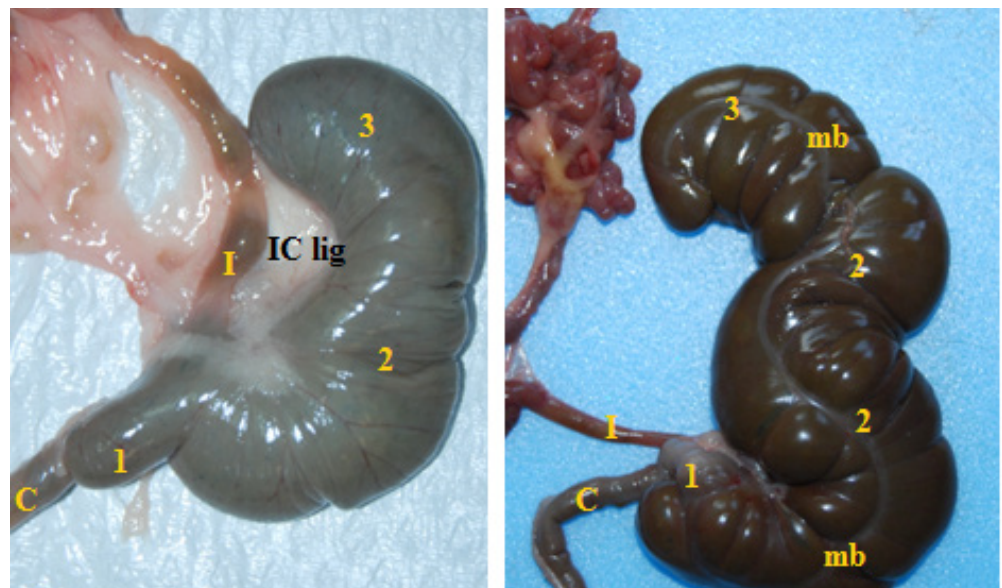

Figure 2. The cecum pattern in rat - left and guinea pig - right. The comma shape cecum of the rat lacking muscular bands and haustra. The coiled shape of the guinea pig cecum present three muscular bands.

$\mathrm{Mb}$ - ventral muscular band. 1 - Ampula ceci; 2 - Corpus ceci; 3 - Apex ceci.

$\mathrm{C}$ - Ascendant ansa of the colon; I - ileum. IC - ileocecal ligament.

In the ratan extension from theradixmesenterii connect the ileum with the apex ceci. The ampulla and proximal colon were connected with the distal ileum by a mesenterial ligament (Snipes, 1981). Both connection elements strengthen the curved cecum of the rat. In guinea pig, the mesenterial connections were made by mesenterial plicae: plica caecocolica, plica ileocolica (Figure 2).

\section{Conclusions}

The cecum macroscopic anatomy in rat and guinea pig show both similarities and differences. The similarities are related to the ventral abdomi- nal topography and the differences are related to the presence of a smooth simple cecum in rat compare to the coliled, haustrated pattern in guinea pig.

Acknowledgments. This research did not receive any specific grant from funding agencies in the public, commercial, or not-for-profit sectors.

\section{References}

1. Snipes RL (1981). Anatomy of the cecum of the laboratory mouse and rat. Anat Embryol (Berl), 162: 455-474.

2. Stan F (2014). Anatomical particularities of the cecum in rabbits and chinchillas. BUSAMV-CN, 71: 406-412. 